TRANSACTIONS OF THE

AMERICAN MATHEMATICAL SOCIETY

Volume 363, Number 9, September 2011, Pages 4929-4944

S 0002-9947(2011)05296-7

Article electronically published on March 4, 2011

\title{
ON THE POINTWISE IMPLEMENTATION OF NEAR-ACTIONS
}

\author{
ASGER TÖRNQUIST
}

\begin{abstract}
We show that the continuum hypothesis implies that every measure preserving near-action of a group on a standard Borel probability space $(X, \mu)$ has a pointwise implementation by Borel measure preserving automorphisms.
\end{abstract}

\section{INTRODUCTION}

(A) Let $(X, \mu)$ be a standard non-atomic Borel probability space. A Borel bijection $f: X \rightarrow X$ is measure preserving (m.p.) if for all Borel $A \subseteq X, \mu\left(f^{-1}(A)\right)=$ $\mu(A)$. In this paper the group of measure preserving Borel bijections on $(X, \mu)$ will be denoted by $G_{\mathrm{mp}}(X, \mu)$. Let

$$
I_{\mathrm{mp}}(X, \mu)=\left\{f \in G_{\mathrm{mp}}(X, \mu): f(x)=x \text { a.e. }\right\},
$$

that is, $I_{\mathrm{mp}}(X, \mu)$ is the (normal) subgroup of all those measure preserving bijections that fix all but a null set of points in $X$. The cosets in

$$
G_{\mathrm{mp}}(X, \mu) / I_{\mathrm{mp}}(X, \mu)
$$

are usually called measure preserving transformations, and they form a group denoted by $\operatorname{Aut}(X, \mu)$. The group $\operatorname{Aut}(X, \mu)$ is a Polish group when equipped with the weak topology, i.e. the topology it inherits when naturally identified with a subgroup of the unitary group of $L^{2}(X, \mu)$; see 9 . We will denote by $\bar{\varphi}$ the canonical homomorphism of $G_{\mathrm{mp}}(X, \mu)$ onto $\operatorname{Aut}(X, \mu)$.

The present paper is concerned with the following fundamental question:

Question 1.1 ("The lifting problem for $\operatorname{Aut}(X, \mu)$ "). Is it possible to find a homomorphism $h: \operatorname{Aut}(X, \mu) \rightarrow G_{\mathrm{mp}}(X, \mu)$ such that $\bar{\varphi} \circ h(T)=T$ for all measure preserving transformations $T$ ? That is, does the identity automorphism $\operatorname{Id}: \operatorname{Aut}(X, \mu) \rightarrow \operatorname{Aut}(X, \mu)$ "split",

$$
\operatorname{Id}: \operatorname{Aut}(X, \mu) \stackrel{h}{\longrightarrow} G_{\mathrm{mp}}(X, \mu) \stackrel{\bar{\varphi}}{\longrightarrow} \operatorname{Aut}(X, \mu) \text {. }
$$

Received by the editors October 4, 2009 and, in revised form, January 19, 2010.

2010 Mathematics Subject Classification. Primary 03E15, 37A05.

Key words and phrases. Ergodic theory, near-actions, spatial actions, descriptive set theory.

This research was supported by the Austrian Science Foundation FWF grant no. P19375-N18.

$$
\begin{array}{r}
\text { (C)2011 American Mathematical Society } \\
\text { Reverts to public domain } 28 \text { years from publication }
\end{array}
$$


We will call such a map $h$ a (homomorphic) lifting of the identity automorphism on $\operatorname{Aut}(X, \mu)$. Our main result is:

Theorem 1.2. Assume that the Continuum Hypothesis, $\mathrm{CH}$, holds. Then the identity automorphism $\operatorname{Id}: \operatorname{Aut}(X, \mu) \rightarrow \operatorname{Aut}(X, \mu)$ splits, that is, there is a homomorphic lifting $h: \operatorname{Aut}(X, \mu) \rightarrow G_{\mathrm{mp}}(X, \mu)$ such that $\bar{\varphi} \circ h=\mathrm{Id}$.

(B) The motivation behind Question 1.1 comes from ergodic theory, where the group $\operatorname{Aut}(X, \mu)$ is of fundamental interest. In the study of measure preserving dynamical systems, an important distinction is made between spatial actions on the one hand, and near-actions on the other hand. A spatial measure preserving action of a group $G$ is simply an action $\rho: G \times X \rightarrow X$ of $G$ on $X$ (sometimes written $\rho: G \curvearrowright X)$ in the usual sense, that additionally preserves the measure, i.e. for all Borel $B \subseteq X$ we have

$$
\mu(B)=\mu(\rho(g)(B)) .
$$

Here it is part of our assumption on $\rho$ that $\rho(g)(B)$ is $\mu$-measurable for each $g \in G$ and Borel $B \subseteq X$. If we additionally assume that $\rho(g, \cdot): x \mapsto \rho(g, x)$ is a Borel function for each $g \in G$ (that is, $\rho$ corresponds to a homomorphism of $G$ into $G_{\mathrm{mp}}(X, \mu)$ ), then we will call $\rho$ a spatial action by (measure preserving) Borel automorphisms.

In most cases of interest $G$ will be a Polish group, or at least a topological group, in which case it may be natural to further require that $\rho: G \times X \rightarrow X$ be Borel. We call such an action $\rho$ a Borel spatial action (following the conventions of [7]).

On the other hand, a near-action of $G$ is a map $\eta: G \times X \rightarrow X$ such that

(1) If $e \in G$ is the identity element, then $\eta(e)(x)=x$ for almost all $x$.

(2) For all $g, h \in G, \eta(g h)(x)=\eta(g)(\eta(h)(x))$ for almost all $x$.

(3) For all Borel $B \subseteq X$ and $g \in G, \mu(B)=\mu(\eta(g)(B))$.

Again, it is part of the definition of a near-action that $\eta(g)(B)$ is measurable for all $g \in G$. If $G$ is a topological group we call $\eta$ a measure preserving Borel near-action if $\eta$ is Borel as a function $\eta: G \times X \rightarrow X$.

The above notions of spatial action and near-action and the related concepts may be generalized to their measure class preserving counterparts in the obvious way.

There is a direct correspondence between the near-actions of a group $G$ and the homomorphisms of $G$ into $\operatorname{Aut}(X, \mu)$ : Each element of $g \in G$ defines through $x \mapsto \eta(g)(x)$ a measurable m.p. bijection almost everywhere, and thus an element of $T_{g} \in \operatorname{Aut}(X, \mu)$. Condition (2) of the definition of a near-action ensures that $g \mapsto T_{g}$ is a homomorphism, which is uniquely determined by $\eta$. If on the other hand $f: G \rightarrow \operatorname{Aut}(X, \mu)$ is a homomorphism, we obtain a near-action of $G$ by picking a representative in $G_{\mathrm{mp}}(X, \mu)$ for $f(g)$, for each $g \in G$. There are of course many near-actions corresponding to $f$.

If $\eta: G \times X \rightarrow X$ is a near-action, we call a (Borel) spatial action $\rho: G \times X \rightarrow X$ a (Borel) spatial model of $\eta$ if

$$
\eta(g, x)=\rho(g, x) \mu \text { a.e. } x .
$$

The relationship between homomorphisms and liftings on the one hand, and nearactions and Borel spatial models on the other hand is clear: If $\eta: G \times X \rightarrow X$ is a near-action, and $f: G \rightarrow \operatorname{Aut}(X, \mu)$ is the corresponding homomorphism, then 
$\eta$ has a spatial model if and only if there is a homomorphism $h: G \rightarrow G_{\mathrm{mp}}(X, \mu)$ such that

$$
\bar{\varphi} \circ h=f .
$$

Thus similarly to Question 1.1 we may ask:

Question 1.3. If $\eta: G \times X \rightarrow X$ is an m.p. near-action of a group $G$, does $\eta$ have a spatial model $\rho$ acting by Borel automorphisms?

If above we require that $\eta$ be a Borel near-action, and ask for a Borel spatial model, this is a well-studied question which has a negative solution in general: It was shown by Glasner, Tsirelson and Weiss [7] that there are Polish groups which do not admit any non-trivial m.p. Borel spatial actions. A particular instance of this is the group $\operatorname{Aut}(X, \mu)$ itself, but Glasner, Tsirelson and Weiss' results encompass all so-called Levy groups; see [7] and [8. On the other hand, a classical theorem of Mackey [14] shows that if $G$ is a locally compact group, then any Borel m.p. near-action of $G$ has a Borel spatial model. This result was recently extended by Kwiatkowska and Solecki 13 to apply to all isometry groups of locally compact metric spaces.

However, in sharp contrast to the Glasner-Tsirelson-Weiss result, we will show the following:

Theorem 1.4. Suppose $G$ is a group of cardinality at most $\aleph_{1}$. Then every m.p. near-action of $G$ on a standard Borel probability space $(X, \mu)$ has a spatial model which acts by Borel m.p. automorphisms. Equivalently, if $f: G \rightarrow \operatorname{Aut}(X, \mu)$ is a homomorphism, then there is a homomorphism $h: G \rightarrow G_{\mathrm{mp}}(X, \mu)$ such that $\bar{\varphi} \circ h=f$. In particular, if $C H$ holds, then all m.p. near-actions have a spatial model acting by m.p. Borel automorphisms.

It is of course a consequence of the Glasner-Tsirelson-Weiss result that the nearaction in the previous theorem is in general not a Borel action when $G$ is a Polish group.

Theorem 1.2 is a direct consequence of Theorem 1.4. Theorem [1.4 holds more generally for measure class preserving near-actions; see (C) below.

It is natural to also consider the easier question of simply having a (measurable) spatial model of a near-action. Here it turns out that $\mathrm{CH}$ can be replaced with Martin's Axiom:

Theorem 1.5. Assume that Martin's Axiom, MA, holds. Then every m.p. nearaction on a standard Borel probability space has a spatial model.

(C) After reading a note circulated by this author early in the summer of 2009 and containing the proof of Theorem 1.2, David Fremlin was kind enough to point out to the author that his proof shows something much more general than Theorem 1.2 .

Indeed, let $X$ be a standard Borel space, and let $\mathcal{B}(X)$ denote the $\sigma$-algebra of Borel sets on $X$. Let $\mathcal{J}$ be an ideal in $\mathcal{B}(X)$. Then define $G(X, \mathcal{J})$ to be the group consisting of those Borel bijections $f: X \rightarrow X$ such that $f^{-1}(B) \in \mathcal{J}$ if and only if $B \in \mathcal{J}$, for all $B \in \mathcal{J}$. Then we may form the group

$$
I(X, \mathcal{J})=\{f \in G(X, \mathcal{J}):\{x \in X: f(x) \neq x\} \in \mathcal{J}\} .
$$

Clearly $I(X, \mathcal{J})$ is a normal subgroup of $G(X, \mathcal{J})$, and so we may consider the quotient group

$$
\operatorname{Aut}(X, \mathcal{J})=G(X, \mathcal{J}) / I(X, \mathcal{J})
$$


Denote by $\varphi_{\mathcal{J}}: G(X, \mathcal{J}) \rightarrow \operatorname{Aut}(X, \mathcal{J})$ the corresponding homomorphism with kernel $I(X, \mathcal{J})$. Then Theorem 1.4 has the following generalization:

Theorem 1.6. Suppose $\mathcal{J}$ is a $\sigma$-ideal in $\mathcal{B}(X)$ such that there is at least one uncountable Borel set $B \in \mathcal{J}$. Then if $G \leq \operatorname{Aut}(X, \mathcal{J})$ is any subgroup at most of cardinality $\aleph_{1}$, then there is a homomorphism $h: G \rightarrow G(X, \mathcal{J})$ such that $\varphi_{\mathcal{J}} \circ h=$ $\operatorname{Id}_{G}$.

If we take $\mathcal{J}$ to be the $\sigma$-ideal of $\mu$-null sets of some standard Borel probability measure on $X$, then we obtain the obvious generalization of Theorem 1.4 to measure class preserving near actions and spatial actions. Another case of interest where Theorem [1.6 applies is if we take $X$ to be a Polish space, and let $\mathcal{J}$ be the ideal of meagre sets.

(D) Question 1.1 belongs to a line of set-theoretic research into quotient structures and their homomorphisms. It is at least superficially related to the lifting

problem for the measure algebra: Recall that if $\mathcal{B}(X)$ is the set of Borel subsets of some standard Borel probability space $(X, \mu)$, and $\mathcal{I}(X)$ is the ideal of $\mu$ null sets in $\mathcal{B}(X)$, then

$$
\operatorname{MALG}(X, \mu)=\mathcal{B}(X) / \mathcal{I}(X) .
$$

Let $\tilde{\varphi}: \mathcal{B}(X) \rightarrow \operatorname{MALG}(X, \mu)$ be the canonical Boolean algebra homomorphism with kernel $\mathcal{I}(X)$. It was shown by von Neumann and Stone in 22 that under $\mathrm{CH}$ there is a Boolean algebra homomorphism $h: \operatorname{MALG}(X, \mu) \rightarrow \mathcal{B}(X)$ (a "lifting") such that $\tilde{\varphi} \circ h$ is the identity on $\operatorname{MALG}(X, \mu)$, i.e., that the identity homomorphism Id : $\operatorname{MALG}(X, \mu) \rightarrow \operatorname{MALG}(X, \mu)$ 'splits' over $\tilde{\varphi}$. Later Shelah famously showed in 18 that it is consistent with ZFC and $2^{\aleph_{0}}=\aleph_{2}$ that Id : $\operatorname{MALG}(X, \mu) \rightarrow$ $\operatorname{MALG}(X, \mu)$ does not split. On the other hand, Carlson, Frankiewicz and Zbierski showed in [1] that it is consistent with $2^{\aleph_{0}}=\aleph_{2}$ that there is a lifting.

There have been many other interesting results regarding the structure of quotient objects, perhaps the most well known being the study of isomorphisms of the Boolean algebras $\mathcal{P}(\omega) /$ FIN and $\mathcal{P}(\omega) / I$ for $I$ an analytic ideal; see [19, 20], 21], [2] and [3. Some remarkable recent results regarding a similar problem for the automorphisms of the Calkin Algebra $\mathcal{C}(H)=\mathcal{B}(H) / \mathcal{K}(H)$, where $H$ is an infinite dimensional separable Hilbert space and $\mathcal{K}(H)$ is the ideal of compact operators in the $C^{*}$-algebra of all bounded operators $\mathcal{B}(H)$, have been achieved by Phillips and Weaver [17, and by Ilijas Farah in [4.

The results quoted above regarding the lifting problem for $\operatorname{MALG}(X, \mu)$ suggest several similar problems for the lifting problem for $\operatorname{Aut}(X, \mu)$. In the last section of this paper we discuss these and other open problems that promise to be quite interesting for future research.

(E) The paper is organized into four sections, including the introduction. $\S 2$ is dedicated to the proof of Theorem 1.6. In $\S 3$ we prove Theorem 1.5, that assuming Martin's Axiom there is a Lebesgue measurable lifting of $\operatorname{Aut}(X, \mu)$. Finally, in $\S 4$ we discuss several open questions related to the theme of this paper.

\section{Proof of the MAin theorem}

In this section we will prove Theorem 1.6 from which Theorems 1.2 and 1.4 follow. We fix once and for all a standard Borel space $X$ and a $\sigma$-ideal $\mathcal{J}$ in $\mathcal{B}(X)$. Denote by $\operatorname{co}(\mathcal{J})$ the filter of all $Z \in \mathcal{B}(X)$ such that $X \backslash Z \in \mathcal{J}$. Recall 
that $\varphi_{\mathcal{J}}: G(X, \mathcal{J}) \rightarrow \operatorname{Aut}(X, \mathcal{J})$ denotes the canonical epimorphism with kernel $I(X, \mathcal{J})$.

The proof of Theorem 1.6 requires several lemmata. As a point of departure let us note the following easy fact:

Lemma 2.1. Let $H<\operatorname{Aut}(X, \mathcal{J})$ be a countable group, and let $T_{0} \in \operatorname{Aut}(X, \mathcal{J}) \backslash$ $H$. Suppose $\rho: H \times X \rightarrow X$ is an action by Borel automorphisms such that $\varphi_{\mathcal{J}}(\rho(T, \cdot))=T$ for all $T \in H$. If $\varphi_{\mathcal{J}}\left(\theta_{0}\right)=T_{0}$, then there is a Borel set $Z \subseteq X$ such that $Z \in \operatorname{co}(\mathcal{J}), Z$ is invariant under $\rho$ and $\theta_{0}$, and there is an action $\tilde{\rho}$ : $\left\langle H \cup\left\{T_{0}\right\}\right\rangle \times Z \rightarrow Z$ such that $\tilde{\rho}\left(T_{0}, \cdot\right)=\theta_{0}\lceil Z$ and $\tilde{\rho}\lceil H \times Z=\rho \uparrow H \times Z$.

Proof. Let $Z$ be the set of all $x \in X$ such that for all $n_{1}, \ldots, n_{k} \in\{-1,1\}$, $n_{0}, n_{k+1} \in\{-1,0,1\}$ and $\gamma_{1}, \ldots, \gamma_{k} \in H$ it holds that if

$$
T_{0}^{n_{0}} \gamma_{1} T_{0}^{n_{1}} \cdots \gamma_{k} T_{0}^{n_{k+1}}=\mathrm{Id},
$$

then

$$
\theta_{0}^{n_{0}} \rho\left(\gamma_{1}, \cdot\right) \theta_{0}^{n_{1}} \cdots \rho\left(\gamma_{k}, \cdot\right) \theta_{0}^{n_{k+1}}(x)=x .
$$

Clearly $Z$ is Borel, $Z \in \operatorname{co}(\mathcal{J})$ and $Z$ is invariant under $\rho$ and $\theta_{0}$. For $z \in Z$ and $T \in\left\langle H \cup\left\{T_{0}\right\}\right\rangle$ write $T=T_{0}^{n_{0}} \gamma_{1} T_{0}^{n_{1}} \cdots \gamma_{k} T_{0}^{n_{k+1}}$, with $\gamma_{1}, \ldots, \gamma_{k} \in H$, and define

$$
\tilde{\rho}(T, z)=\theta_{0}^{n_{0}} \rho\left(\gamma_{1}, \cdot\right) \theta_{0}^{n_{1}} \cdots \rho\left(\gamma_{k}, \cdot\right) \theta_{0}^{n_{k+1}}(z) .
$$

If $T=T_{0}^{n_{0}^{\prime}} \gamma_{1}^{\prime} T_{0}^{n_{1}^{\prime}} \cdots \gamma_{l}^{\prime} T_{0}^{n_{l+1}^{\prime}}$ is another representation of $T$ of this form, then by the definition of $Z$ we must have

$$
\tilde{\rho}(T, z)=\theta_{0}^{n_{0}^{\prime}} \rho\left(\gamma_{1}^{\prime}, \cdot\right) \theta_{0}^{n_{1}^{\prime}} \cdots \rho\left(\gamma_{l}^{\prime}, \cdot\right) \theta_{0}^{n_{l+1}^{\prime}}(z)
$$

Thus $\tilde{\rho}$ is well-defined on $Z$. Clearly $\tilde{\rho}$ is an $\left\langle H \cup\left\{T_{0}\right\}\right\rangle$-action by Borel bijections such that $\tilde{\rho}\left(T_{0}, \cdot\right)=\theta_{0}\lceil Z$ and $\tilde{\rho}\lceil H \times Z=\rho \uparrow H \times Z$.

If the conclusion of Lemma 2.1 were always true for $Z=X$ and for some $\theta_{0}$ with $\varphi_{\mathcal{J}}\left(\theta_{0}\right)=T_{0}$, then Theorem [1.6 would follow from an easy transfinite induction. However, we certainly cannot expect to have Lemma 2.1 with $Z=X$ for just any $\theta_{0}$ with $\varphi_{\mathcal{J}}\left(\theta_{0}\right)=T_{0}$ if we proceed naïvely, loosely speaking since the action of $H$ might behave very differently on some $\mathcal{J}$ set than on a given $\operatorname{co}(\mathcal{J})$ set.

We will prove a lemma that shows that, under certain conditions, there is always some $\theta_{0}$ with $\varphi_{\mathcal{J}}\left(\theta_{0}\right)=T_{0}$ that satisfies Lemma 2.1 with $Z=X$. First we need to recall the notion of a (Bernoulli) shift action, and a lemma regarding their universality among Borel actions of countable groups. If $H \leq G$ are countable groups and $X$ is a standard Borel space, recall that a Borel action $\beta: H \curvearrowright X^{G}$ is defined by

$$
(\beta(h)(x))(g)=x\left(h^{-1} g\right)
$$

for $h \in H$ and $g \in G$.

Lemma 2.2 (Folklore). Let $H \leq G$ be countable groups and let $X=2^{\mathbb{N}}=\{0,1\}^{\mathbb{N}}$ be a Cantor space. Then the shift action $\beta: H \curvearrowright X^{G}$ has the following universality property: If $Y$ is a standard Borel space and $\sigma: H \curvearrowright Y$ is a Borel action of $H$ on $Y$, then there is a Borel injection $\psi: Y \rightarrow X^{G}$ such that for all $h \in H$,

$$
\psi(\sigma(h)(x))=\beta(h)(\psi(x)),
$$

i.e., $\psi$ conjugates the actions $\sigma$ and $\beta \mid \psi(Y)$. 
Proof. Since $X^{H}$ may be naturally identified with a subset of $X^{G}$, we may assume that $H=G$. Let $\left(B_{n}\right)_{n \in \mathbb{N}}$ be a sequence of Borel sets that separates the points of $Y$. Define $\psi: Y \rightarrow X^{H}$ by

$$
\psi(x)(h)(n)= \begin{cases}1 & \text { if } \sigma\left(h^{-1}\right)(x) \in B_{n} \\ 0 & \text { otherwise }\end{cases}
$$

Then

$$
\begin{aligned}
& \psi(\sigma(g)(x))(h)(n)=1 \Longleftrightarrow \sigma\left(h^{-1} g\right)(x) \in B_{n} \Longleftrightarrow \\
& \psi(x)\left(g^{-1} h\right)(n)=1 \Longleftrightarrow \beta(g)(\psi(x))(h)(n)=1,
\end{aligned}
$$

which shows that $\psi$ conjugates the $\sigma$ and $\beta$ actions. Finally, since $\left(B_{n}\right)$ separates points in $Y, \psi$ is $1-1$.

We now have the following strong version of Lemma 2.1, which will play a key role in our construction:

Lemma 2.3 ("Rearrangement"). Let $H \leq G$ be countable groups, and suppose there are countable groups $G_{i}, i \in \mathbb{N}$, such that $G \leq G_{i}$ for all $i$. Let $X$ be a standard Borel space which is partitioned into Borel pieces,

$$
X=X_{0} \sqcup \bigsqcup_{i \in \mathbb{N}}\left(2^{\mathbb{N}}\right)^{G_{i}},
$$

that is, $X$ is the disjoint union of $X_{0}$ and $\left(2^{\mathbb{N}}\right)^{G_{i}}, i \in \mathbb{N}, X_{0}$ is Borel, and $\left(2^{\mathbb{N}}\right)^{G_{i}}$ carries its usual Borel structure for all $i \in \mathbb{N}$. Suppose $\rho: H \curvearrowright X$ is a Borel action of $H$ such that

$$
\rho \uparrow H \times\left(2^{\mathbb{N}}\right)^{G_{i}}
$$

is the shift action. Then there is a Borel action $\hat{\rho}: G \curvearrowright X$ such that $\hat{\rho}\lceil H \times X=\rho$.

Proof. For convenience, let $X_{i}=\left(2^{\mathbb{N}}\right)^{G_{i}}$, so that $X=\bigsqcup_{i \geq 0} X_{i}$. Note that by definition, $X_{0}$ is $\rho$-invariant. Thus by Lemma 2.2 we may find Borel injections $\psi_{i}: X_{0} \rightarrow\left(2^{\mathbb{N}}\right)^{G_{i}}, i \geq 1$, such that $\psi_{i}$ conjugates $\rho\left\lceil X_{0}\right.$ and $\rho\left\lceil\psi_{i}\left(X_{0}\right)\right.$. We let $\psi_{0}: X_{0} \rightarrow X_{0}$ be $\psi_{0}(x)=x$. Now let

$$
Z_{0}=X_{0} \sqcup\left(X_{1} \backslash \psi_{1}\left(X_{0}\right)\right) \text {, }
$$

and for $i \geq 1$ let

$$
Z_{i}=\left(X_{i+1} \backslash\left(\psi_{i+1}\left(X_{0}\right)\right)\right) \sqcup \psi_{i}\left(X_{0}\right) .
$$

Then each $Z_{i}$ is $\rho$-invariant, and the functions

$$
\bar{\psi}_{i}: Z_{i} \rightarrow X_{i+1}: x \mapsto \begin{cases}\psi_{i+1} \psi_{i}^{-1}(x) & \text { if } x \in \psi_{i}\left(X_{0}\right), \\ x & \text { otherwise }\end{cases}
$$

define a bijection between $Z_{i}$ and $X_{i+1}, i \geq 0$. Moreover, $\bar{\psi}_{i}$ conjugates the actions $\rho\left\lceil Z_{i}\right.$ and $\rho\left\lceil X_{i+1}\right.$. Now for $i \geq 1$ let $\beta_{i}: G \times X_{i} \rightarrow X_{i}$ be the Bernoulli shift action of $G$. For each $i \geq 0$ we may define a $G$-action $\hat{\rho}_{i}: G \times Z_{i} \rightarrow Z_{i}$ by

$$
\hat{\rho}_{i}(\gamma, x)=\bar{\psi}_{i}^{-1}\left(\beta_{i}\left(\gamma, \bar{\psi}_{i}(x)\right)\right) .
$$

Since $\beta_{i} \uparrow H \times X_{i}=\rho \uparrow H \times X_{i}$ and $\bar{\psi}_{i}$ conjugates the actions $\rho \uparrow Z_{i}$ and $\rho \uparrow X_{i+1}$, we have that $\hat{\rho}_{i} \uparrow H \times Z_{i}=\rho \uparrow H \times Z_{i}$. Thus if we let

$$
\hat{\rho}=\bigcup_{i \in \mathbb{N}} \hat{\rho}_{i}
$$

then $\hat{\rho}$ is a Borel $G$-action as required. 
2.1. The master actions. Fix a group $H \leq \operatorname{Aut}(X, \mathcal{J})$ of cardinality $\aleph_{1}$. Let $\left(T_{\alpha}: \alpha<\omega_{1}\right)$ be an enumeration of $H$. For $\alpha<\omega_{1}$ define

$$
H_{\alpha}=\left\langle T_{\beta}: \beta<\alpha\right\rangle,
$$

i.e., $H_{\alpha}$ is the subgroup generated by all $T_{\beta}, \beta<\alpha$. By definition we let $H_{0}=\{I\}$, the subgroup containing only the identity in $\operatorname{Aut}(X, \mathcal{J})$.

We now describe a natural Polish space $S_{\alpha}$ of countable groups containing a copy of $H_{\alpha}$, and with a sequence of designated elements that will correspond to the $T_{\beta}$, $\beta<\alpha$.

If $x \in 2^{\mathbb{N} \times \mathbb{N}}$, write $R_{x}$ for the relation

$$
m R_{x} n \Longleftrightarrow x(m, n)=1 .
$$

We let

$$
\mathbf{L O}=\left\{x \in 2^{\mathbb{N} \times \mathbb{N}}: R_{x} \text { is a (strict) linear ordering }\right\} .
$$

Elements of $\mathbf{L O}$ will usually be denoted by $<^{*}$ or something similar, and we will write $m<^{*} n$ to indicate what should more correctly be written as $m R_{<*} n$. Moreover, we will write $m \leq^{*} n$ if $m<^{*} n$ or $m=n$. Let GP denote the set of all groups with underlying set $\mathbb{N}$, i.e.,

$$
\begin{aligned}
\mathbf{G P}=\{ & (f, g, e) \in \mathbb{N}^{\mathbb{N} \times \mathbb{N}} \times \mathbb{N}^{\mathbb{N}} \times \mathbb{N}: \\
& (\forall i, j, k \in \mathbb{N})(f(f(i, j), k)=f(i, f(j, k))) \wedge \\
& (\forall i \in \mathbb{N}) f(i, e)=f(e, i)=i \wedge(\forall i) f(i, g(i))=e\} .
\end{aligned}
$$

Again, it is notationally convenient to denote an element in GP by $G$, and refer to the multiplication in $G$ by ${ }_{G}$, the inverse by ${ }^{-1}$, and the identity element by $e_{G}$.

For $\alpha<\omega_{1}$ let

$$
A_{\alpha}=\left\{\left(<^{*}, n\right) \in \mathbf{L O} \times \mathbb{N}:\left(\left\{k \in \mathbb{N}: k<^{*} n\right\},<^{*}\right) \simeq \alpha\right\},
$$

that is, $\left(<^{*}, n\right) \in A_{\alpha}$ if and only if the initial segment $\left\{k: k<^{*} n\right\}$ is order isomorphic to the ordinal $\alpha$.

Lemma 2.4. The set $A_{\alpha}$ is Borel for all $\alpha<\omega_{1}$.

Proof. By induction on $\alpha$. If $\alpha=\beta+1$, then

$$
\begin{aligned}
& A_{\alpha}=\left\{\left(<^{*}, n\right) \in \mathbf{L O} \times \mathbb{N}:\right.(\exists m)\left(\left(<^{*}, m\right) \in A_{\beta} \wedge\right. \\
&\left.\left.(\forall k) k<^{*} n \Longrightarrow k \leq{ }^{*} m\right)\right\} .
\end{aligned}
$$

If $\alpha$ is a limit, fix $\beta_{i}<\alpha, i \in \mathbb{N}$ such that $\sup _{i \in \mathbb{N}} \beta_{i}=\alpha$. Then

$$
\begin{aligned}
& A_{\alpha}=\left\{\left(<^{*}, n\right) \in \mathbf{L O} \times \mathbb{N}:(\forall i)(\exists m)\left(<^{*}, m\right) \in A_{\beta_{i}} \wedge\right. \\
&\left.(\forall k) k<^{*} n \Longrightarrow(\exists i)(\exists l)\left(<^{*}, l\right) \in A_{\beta_{i}} \wedge k<^{*} l\right\} .
\end{aligned}
$$

Definition. For $\alpha<\omega_{1}$ we define $\mathcal{S}_{\alpha} \subseteq \mathbf{L O} \times \mathbf{G P}$ to consist of all $\left(<^{*}, G\right) \in$ $\mathbf{L O} \times \mathbf{G P}$ such that:

(i) For some $n \in \mathbb{N},\left(<^{*}, n\right) \in A_{\alpha}$.

(ii) There is a monomorphism $\varphi: H_{\alpha} \rightarrow G$ such that $\left(<^{*}, \varphi\left(T_{\beta}\right)\right) \in A_{\beta}$ for all $\beta<\alpha$.

Lemma 2.5. The set $\mathcal{S}_{\alpha}$ is Borel for all $\alpha<\omega_{1}$. 
Proof. We claim that $\left(<^{*}, G\right)$ satisfies (i) and (ii) if and only if the following holds. There is an $n \in \mathbb{N}$ such that

$\left(i^{\prime}\right)\left(<^{*}, n\right) \in A_{\alpha}$.

(ii') For all $\beta_{0}, \ldots, \beta_{l}<\alpha, n_{0}, \ldots, n_{l} \in\{-1,1\}$ and $m_{0}, \ldots, m_{l} \in \mathbb{N}$ it holds that

$$
\text { (a) } \begin{array}{r}
T_{\beta_{0}}^{n_{0}} T_{\beta_{1}}^{n_{1}} \cdots T_{\beta_{l}}^{n_{l}}=I \wedge(\forall i \leq l)\left(<^{*}, m_{i}\right) \in A_{\beta_{i}} \Longrightarrow \\
m_{0}^{n_{0}} \cdot G m_{1}^{n_{1}} \cdots m_{l}^{n_{l}}=e_{G}
\end{array}
$$

and

$$
\text { (b) } \begin{array}{r}
T_{\beta_{0}}^{n_{0}} T_{\beta_{1}}^{n_{1}} \cdots T_{\beta_{l}}^{n_{l}} \neq I \wedge(\forall i \leq l)\left(<^{*}, m_{i}\right) \in A_{\beta_{i}} \Longrightarrow \\
m_{0}^{n_{0}} \cdot G m_{1}^{n_{1}} \cdots m_{l}^{n_{l}} \neq e_{G} .
\end{array}
$$

It is clear that $(i)$ and $(i i)$ imply $\left(i^{\prime}\right)$ and $\left(i i^{\prime}\right)$, and that $\left(i^{\prime}\right)$ and $\left(i i^{\prime}\right)$ are Borel conditions. To show that $\left(i^{\prime}\right)$ and $\left(i i^{\prime}\right)$ imply $(i)$ and $(i i)$, it suffices to show that if $\left(<^{*}, G\right)$ satisfies $\left(i^{\prime}\right)$ and $\left(i i^{\prime}\right)$, then the map $\varphi: H_{\alpha} \rightarrow G$, given by

$$
\begin{aligned}
& \varphi(x)=m_{0}^{n_{0}} \cdot G m_{1}^{n_{1}} \cdots m_{l}^{n_{l}} \Longleftrightarrow \\
& x=T_{\beta_{0}}^{n_{0}} T_{\beta_{1}}^{n_{1}} \cdots T_{\beta_{l}}^{n_{l}} \wedge(\forall i \leq l)\left(<^{*}, m_{0}\right) \in A_{\beta_{i}},
\end{aligned}
$$

defines a monomorphism with $\varphi\left(T_{\beta}\right)=m$ if and only if $(<*, m) \in A_{\beta}$. That $\varphi$ is a well-defined function follows easily from (a). If $\varphi(x)=e_{G}$ and

$$
x=T_{\beta_{0}}^{n_{0}} T_{\beta_{1}}^{n_{1}} \cdots T_{\beta_{l}}^{n_{l}},
$$

then $x=I$ follows from (b). Thus $\varphi$ is a monomorphism as required.

Remark. For $\left(<^{*}, G\right) \in \mathcal{S}_{\alpha}$, the unique monomorphism $\varphi: H_{\alpha} \rightarrow G$ satisfying (ii) in the definition of $\mathcal{S}_{\alpha}$ will be called the canonical monomorphism $H_{\alpha} \rightarrow G$.

Definition. For $\alpha<\omega_{1}$, the $\alpha$ 'th master action $\sigma_{\alpha}: H_{\alpha} \curvearrowright \mathcal{M}_{\alpha}$ is defined by

(1) $\mathcal{M}_{\alpha}=\mathcal{S}_{\alpha} \times\left(2^{\mathbb{N}}\right)^{\mathbb{N}}$;

(2) for $\beta<\omega_{1}$,

$$
\begin{aligned}
& \sigma_{\alpha}\left(T_{\beta}\right)\left(<_{0}^{*}, G_{0}, x\right)=\left(<_{1}^{*}, G_{1}, y\right) \Longleftrightarrow \\
& \quad<_{0}^{*}=<_{1}^{*} \wedge G_{0}=G_{1} \wedge \\
& (\forall m)\left(\left(<_{0}^{*}, m\right) \in A_{\beta} \Longrightarrow(\forall n) y(n)=x\left(m^{-1} \cdot G_{0} n\right)\right) .
\end{aligned}
$$

Note that $\sigma_{\alpha}\left\lceil\left\{\left(<^{*}, G\right)\right\} \times\left(2^{\mathbb{N}}\right)^{\mathbb{N}}\right.$ is isomorphic to the shift-action of $H_{\alpha}$ on $\left(2^{\mathbb{N}}\right)^{\mathbb{N}}$, when $H_{\alpha}$ is identified canonically with its image under the canonical monomorphism $\varphi: H_{\alpha} \rightarrow G$, and $G$ is identified with its underlying set $\mathbb{N}$. So we think of $\left(2^{\mathbb{N}}\right)^{\mathbb{N}}$ as $\left(2^{\mathbb{N}}\right)^{G}$ and think of $\sigma_{\alpha} \mid\left\{\left(<^{*}, G\right)\right\} \times\left(2^{\mathbb{N}}\right)^{\mathbb{N}}$ as the shift action $H_{\alpha} \curvearrowright\left(2^{\mathbb{N}}\right)^{G}$ for each $\left(<^{*}, G\right) \in \mathcal{S}_{\alpha}$. It is clear that condition (2) of the definition of $\sigma_{\alpha}$ defines the action uniquely since the $T_{\beta}, \beta<\alpha$ generate $H_{\alpha}$. Moreover, $\sigma_{\alpha}\left(T_{\beta}\right)$ is a Borel automorphism of $\mathcal{M}_{\alpha}$ for each $\beta<\alpha$, and so $\sigma_{\alpha}$ defines a Borel action of $H_{\alpha}$ on $\mathcal{M}_{\alpha}$. Finally note that if $\beta<\alpha$, then $\mathcal{M}_{\alpha} \subseteq \mathcal{M}_{\beta}$ and that for the actions $\sigma_{\alpha}, \sigma_{\beta}$ we have

$$
\sigma_{\alpha}\left\lceil H_{\beta} \times \mathcal{M}_{\alpha}=\sigma_{\beta}\left\lceil H_{\beta} \times \mathcal{M}_{\alpha}\right.\right.
$$


where again $H_{\beta}$ is canonically identified with a subgroup of $G$ for each $\left(<^{*}, G\right) \in$ $\mathcal{S}_{\alpha} \subseteq \mathcal{S}_{\beta}$. The following is clear from the definitions:

Lemma 2.6. If $\alpha<\omega_{1}$ is a limit ordinal, it holds for the master action $\sigma_{\alpha}$ that

$$
\sigma_{\alpha}=\bigcup_{\beta<\alpha} \sigma_{\beta} \uparrow \mathcal{M}_{\alpha}
$$

We are now ready to prove Theorem 1.6. The idea is to construct the homomorphism $h: H \rightarrow G(X, \mathcal{J})$ by transfinite induction on $\omega_{1}$ by constructing in stages $h_{\alpha}: H_{\alpha} \rightarrow G(X, \mathcal{J})$, at each stage making sure that on some $\mathcal{J}$ set we have a large invariant "reservoir" of master actions. This will allow us to use Lemma 2.3 to extend the homomorphism at each stage of the induction.

Proof of Theorem 1.6. Since $\mathcal{J}$ contains an uncountable Borel set we can assume that $X$ has the form $X=X_{0} \sqcup 2^{\mathbb{N}} \times \mathcal{M}_{0}$ (disjoint union) such that $X_{0} \in \operatorname{co}(\mathcal{J})$. We construct by induction on $\alpha<\omega_{1}$ homomorphisms $h_{\alpha}: H_{\alpha} \rightarrow G(X, \mathcal{J})$ and uncountable Borel sets $Y_{\alpha} \subseteq 2^{\mathbb{N}}$ such that:

(1) $h_{0}(I)=\mathrm{Id}, Y_{0}=2^{\mathbb{N}}$.

(2) $h_{\alpha}: H_{\alpha} \rightarrow G(X, \mathcal{J})$ is a homomorphism such that $\varphi_{\mathcal{J}}\left(h_{\alpha}(T)\right)=T$ for all $T \in H_{\alpha}$.

(3) If $\beta<\alpha$, then $Y_{\beta} \supseteq Y_{\alpha}$ and $Y_{\beta} \backslash Y_{\alpha}$ is countable.

(4) For $(y, x) \in Y_{\alpha} \times \mathcal{M}_{\alpha}$ we have $h_{\alpha}(T)(y, x)=\left(y, \sigma_{\alpha}(T)(x)\right)$ for all $T \in H_{\alpha}$.

(5) If $\beta<\alpha$, then $h_{\beta}=h_{\alpha}\left\lceil H_{\beta}\right.$.

If this can be done, then we let

$$
h=\bigcup_{\alpha<\omega_{1}} h_{\alpha}
$$

By condition (5), $h$ is a function, $h: H \rightarrow G(X, \mathcal{J})$. By condition $(2)$ it is a homomorphism with $\varphi_{\mathcal{J}}(h(T))=T$ for all $T \in H$. Thus to prove Theorem 1.6] it suffices to construct $h_{\alpha}$ and $Y_{\alpha}$ satisfying (1)-(5).

Case 1. $\alpha$ is a limit ordinal.

We let $h_{\alpha}=\bigcup_{\beta<\alpha} h_{\beta}$ and $Y_{\alpha}=\bigcap_{\beta<\alpha} Y_{\beta}$. (1)-(3) and (5) are clearly satisfied. (4) follows by Lemma 2.6.

Case 2. $\alpha=\beta+1$.

Let $\rho_{\beta}: H_{\beta} \times X \rightarrow X$ be the action corresponding to $h_{\beta}$,

$$
\rho_{\beta}(T, x)=h_{\beta}(T)(x) .
$$

By Lemma 2.1 we may find a Borel $Z \subseteq X_{0}$ and $\theta: Z \rightarrow Z$ Borel such that $Z \in$ $\operatorname{co}(\mathcal{J})$ and $\varphi_{\mathcal{J}}(\theta)=T_{\beta}$ (more precisely, an extension $\bar{\theta}$ of $\theta$ satisfies $\varphi_{\mathcal{J}}(\bar{\theta})=T_{\beta}$ ), such that $Z$ is invariant under

$$
\left\{h_{\beta}(T): T \in H_{\beta}\right\} \cup\{\theta\},
$$

and $\rho_{\beta}\left\lceil Z\right.$ extends to an action $\rho^{0}: H_{\alpha} \times Z \rightarrow Z$ such that

$$
\rho^{0} \uparrow H_{\beta} \times Z=\rho_{\beta} \uparrow H_{\beta} \times Z,
$$

and $\rho^{0}\left(T_{\beta}, \cdot\right)=\theta$. 
Pick a countable sequence $\left(y_{i} \in Y_{\beta}: i \in \mathbb{N}\right)$ of distinct elements in $Y_{\beta}$. Let $Y_{\alpha}=Y_{\beta} \backslash\left\{y_{i}: i \in \mathbb{N}\right\}$, and let

$$
W=X \backslash\left(Z \cup Y_{\alpha} \times \mathcal{M}_{\alpha}\right) .
$$

Also pick a sequence $\left(<_{i}^{*}, G_{i}\right) \in \mathcal{S}_{\alpha}, i \in \mathbb{N}$, distinct. Then

$$
W=\left(W \backslash \bigcup_{i \in \mathbb{N}}\left\{\left(y_{i},<_{i}^{*}, G_{i}\right)\right\} \times\left(2^{\mathbb{N}}\right)^{\mathbb{N}}\right) \sqcup \bigsqcup_{i \in \mathbb{N}}\left\{\left(y_{i},<_{i}^{*}, G_{i}\right)\right\} \times\left(2^{\mathbb{N}}\right)^{\mathbb{N}}
$$

is a decomposition of $W$ into $\rho_{\beta}$-invariant pieces. Note that $\rho_{\beta}\left\lceil\left\{\left(y_{i},<_{i}^{*}, G_{i}\right)\right\} \times\right.$ $\left(2^{\mathbb{N}}\right)^{\mathbb{N}}$ is isomorphic to a shift-action of $H_{\beta}<G_{i}$ on $\left(2^{\mathbb{N}}\right)^{G_{i}}$ and by construction $H_{\beta}<H_{\alpha} \leq G_{i}$. Thus the hypothesis of Lemma 2.3 is satisfied for $\rho_{\beta}\left\lceil H_{\beta} \times W\right.$, and so we may find a Borel action $\rho^{1}: H_{\alpha} \times W \rightarrow W$ such that $\rho^{1} \uparrow H_{\beta} \times W=$ $\rho_{\beta}\left\lceil H_{\beta} \times W\right.$.

Finally, we define $\rho^{2}: H_{\alpha} \times\left(Y_{\alpha} \times \mathcal{M}_{\alpha}\right) \rightarrow Y_{\alpha} \times \mathcal{M}_{\alpha}$ by

$$
\rho^{2}(T,(y, x))=\left(y, \sigma_{\alpha}(T)(x)\right) .
$$

Note that by (4), $\rho^{2}$ is an extension of $\rho_{\beta}\left\lceil H_{\beta} \times\left(Y_{\alpha} \times \mathcal{M}_{\alpha}\right)\right.$. It follows that

$$
\rho_{\alpha}=\rho^{0} \cup \rho^{1} \cup \rho^{2}
$$

defines a Borel action of $H_{\alpha}$ extending $\rho_{\beta}$. Let

$$
h_{\alpha}: H_{\alpha} \rightarrow G(X, \mathcal{J}): T \mapsto \rho_{\alpha}(T, \cdot) .
$$

Conditions (1), (3) and (5) are clearly satisfied. (2) follows since $\rho^{0}\left(T_{\beta}, \cdot\right)=\theta$. (4) follows directly from the definition of $\rho^{2}$. Thus the proof of Theorem [1.6 is complete.

Remark. Let $\mu$ be a Borel measure on $X$ and let $\mathcal{J}$ be the ideal in $\mathcal{B}(X)$ of measure zero sets. Then

$$
\operatorname{Aut}(X, \mathcal{J})=G(X, \mathcal{J}) / I(X, \mathcal{J})
$$

is the group of measure class preserving transformations on $(X, \mu)$. If $\mu$ is a $\sigma$-finite measure, then this is a Polish group, usually denoted by $\operatorname{Aut}^{*}(X, \mu)$; see [11, 17.46]. The notions of near-actions and spatial actions can be generalized in the obvious way to measure class preserving actions, and Theorem [1.6 applies to $\operatorname{Aut}^{*}(X, \mu)$. Thus we have:

Corollary 2.7. Let $X$ be a standard Borel space and $\mu$ a Borel measure with at least one uncountable null set (e.g. $X$ uncountable and $\mu$ a $\sigma$-finite measure). Let $G$ be a group of at most cardinality $\aleph_{1}$. Then every measure class preserving near-action of $G$ on $(X, \mu)$ has a spatial model acting by Borel automorphisms.

In particular, if $\mathrm{CH}$ holds, then all measure class preserving near-actions have a spatial model acting by Borel automorphisms.

\section{A Lebesgue measurable lifting From MA}

In this section we consider the easier problem of producing a Lebesgue measurable lifting of Id : $\operatorname{Aut}(X, \mu) \rightarrow \operatorname{Aut}(X, \mu)$, where $(X, \mu)$ denotes a standard Borel probability space. Since it presents no added difficulty in the proof, we will consider the more general problem of producing a lifting of $\operatorname{Id}: \operatorname{Aut}^{*}(X, \mu) \rightarrow \operatorname{Aut}^{*}(X, \mu)$, where $\operatorname{Aut}^{*}(X, \mu)$ denotes the group of measure class preserving automorphisms of $(X, \mu)$. 
Let $G_{\mathrm{LM}}(X, \mu)$ denote the set of all Lebesgue measurable measure class preserving bijections of $(X, \mu)$ which have a Lebesgue measurable inverse, and let

$$
I_{\mathrm{LM}}(X, \mu)=\left\{f \in G_{\mathrm{LM}}(X, \mu): f(x)=x \text { a.e. }\right\} .
$$

Then $I_{\mathrm{LM}}(X, \mu)$ is a subgroup of $G_{\mathrm{LM}}(X, \mu)$ such that

$$
G_{\mathrm{LM}}(X, \mu) / I_{\mathrm{LM}}(X, \mu) \simeq \operatorname{Aut}^{*}(X, \mu),
$$

and we denote by $\varphi_{\mathrm{LM}}: G_{\mathrm{LM}}(X, \mu) \rightarrow \operatorname{Aut}^{*}(X, \mu)$ the canonical epimorphism with kernel $I_{\mathrm{LM}}(X, \mu)$.

It is worthwhile making explicit the similar category version of this. If $Y$ is a topological space, one may consider the group $G_{\mathrm{BM}}(Y)$ of all Baire measurable bijections (see [11, 8.I]) which have a Baire measurable inverse, and which preserve the ideal of meagre sets. Then, in analogy with the above, we may form

$$
I_{\mathrm{BM}}(Y)=\left\{f \in G_{\mathrm{BM}}(Y):\{x \in Y: f(x)=x\} \text { is comeagre }\right\}
$$

and the corresponding quotient group

$$
G_{\mathrm{BM}}(Y) / I_{\mathrm{BM}}(Y)=\operatorname{Aut}_{\mathrm{BM}}(Y) .
$$

Let $\varphi_{\mathrm{BM}}: G_{\mathrm{BM}}(Y) \rightarrow \operatorname{Aut}_{\mathrm{BM}}(Y)$ be the canonical epimorphism.

In this section we prove:

Theorem 3.1. Suppose Martin's Axiom, MA, holds. Then Id : Aut* $(X, \mu) \rightarrow$ $\operatorname{Aut}^{*}(X, \mu)$ splits over $\varphi_{\mathrm{LM}}$, i.e. there is $h: \operatorname{Aut}(X, \mu) \rightarrow G_{\mathrm{LM}}(X, \mu)$ such that $\varphi_{\mathrm{LM}} \circ h=\mathrm{Id}$.

Similarly, assuming $M A$, if $Y$ is a locally compact c.c.c. Hausdorff space (e.g. $Y$ a locally compact Polish space), then $\operatorname{Id}: \operatorname{Aut}_{\mathrm{BM}}(Y) \rightarrow \operatorname{Aut}_{\mathrm{BM}}(Y)$ splits over $\varphi_{\mathrm{BM}}$.

We refer to Chapter II of 12 for background about Martin's Axiom. Theorem 3.1 follows from the following more general statement. Recall that an ideal $\mathcal{I}$ is $\kappa$-additive, where $\kappa$ is a cardinal, if the union of fewer than $\kappa$ sets in $\mathcal{I}$ is again in $\mathcal{I}$. $\mathrm{MA}(\kappa)$ implies that the ideal of Lebesgue null sets and the ideal of meagre sets are $\kappa$-additive; see [12, Theorems 2.21 and 2.22].

Theorem 3.2. Suppose $\kappa \leq 2^{\aleph_{0}}$ and that the ideal of Lebesgue null sets is $\lambda$ additive for all $\lambda<\kappa$. Then for any group $H \leq \operatorname{Aut}^{*}(X, \mu)$ of cardinality $\kappa$ there is a homomorphism $h: H \rightarrow G_{\mathrm{LM}}(X, \mu)$ such that $\varphi_{\mathrm{LM}} \circ h=\operatorname{Id}_{H}$.

A similar result holds for the category case.

Before proving Theorem 3.2, we need two straightforward generalizations of Lemmas 2.1 and 2.2

Lemma 3.3. Let $\kappa<2^{\aleph_{0}}$ and suppose that the ideal of Lebesgue null sets is $\kappa$ additive. Let $H<\operatorname{Aut}^{*}(X, \mu)$ be a group of cardinality $\kappa$, and let $T_{0} \in \operatorname{Aut}^{*}(X, \mu) \backslash$ $H$. Suppose $\rho: H \times X \rightarrow X$ is an action of $H$ by Lebesgue measurable bijections such that $\varphi_{\mathrm{LM}}(\rho(T, \cdot))=T$ for all $T \in H$. If $\theta_{0} \in G_{\mathrm{LM}}(X, \mu)$ is such that $\varphi_{\mathrm{LM}}\left(\theta_{0}\right)=T_{0}$, then there is $Z \subseteq X$ such that $\mu(Z)=1, Z$ is invariant under all $\theta_{0}$ and $\rho(T, \cdot)$ for all $T \in H$, and there is an action $\tilde{\rho}:\left\langle H \cup\left\{T_{0}\right\}\right\rangle \times Z \rightarrow Z$ such that $\tilde{\rho}\left(T_{0}, \cdot\right)=\theta_{0}\lceil Z$ and $\tilde{\rho}\lceil H \times Z=\rho \uparrow H \times Z$.

The same statement holds, mutatis mutandis, in the Baire category case. 
Proof. By the same argument given for Lemma 2.1, the lemma holds for $H$ countable. If $H$ is not countable, then for each countable subgroup $\Delta<H$ we can find $Z_{\Delta} \subseteq X$ with $\mu\left(Z_{\Delta}\right)=1$ and satisfying the lemma for $\Delta$ and $\rho \uparrow \Delta \times X$. Let $\tilde{\rho}_{\Delta}: \Delta \times Z_{\Delta} \rightarrow Z_{\Delta}$ witness this. If we let

$$
Z=\bigcap\left\{Z_{\Delta}: \Delta<H \text { is a countable subgroup }\right\},
$$

then since we assume that the ideal of Lebesgue null sets is $\kappa$-additive, we have $\mu(Z)=1$, and

$$
\tilde{\rho}=\bigcup\left\{\tilde{\rho}_{\Delta} \uparrow \Delta \times Z: \Delta<H \text { is a countable subgroup }\right\}
$$

is as required.

The notion of shift action $\beta: H \curvearrowright X^{G}$ generalizes easily to uncountable groups $H \leq G$. The proof of the following lemma is virtually identical to that of Lemma 2.2 ,

Lemma 3.4. Let $\aleph_{0} \leq \kappa<2^{\aleph_{0}}$, let $H \leq G$ be groups, $|G|=\kappa$, and let $X=2^{\mathbb{N}}$. Then the shift action $\beta: H \curvearrowright X^{G}$ has the following universality property: If $Y$ is a set, $|Y| \leq 2^{\aleph_{0}}$, and $\sigma: H \curvearrowright Y$ is an action of $H$ on $Y$, then there is an injection $\psi: Y \rightarrow X^{G}$ such that for all $h \in H$,

$$
\psi(\sigma(h)(x))=\beta(h)(\psi(x)) .
$$

Proof of Theorem 3.2. We will construct an action $\rho: H \times X \rightarrow X$ by Lebesgue measurable bijections by induction on $\kappa=|H|$ such that

$$
\varphi_{\mathrm{LM}}(\rho(T, \cdot))=T
$$

for all $T \in H$. The proof is made easier compared to the Borel case by the fact that during the inductive construction, we only need to make sure that for each $T \in H, \rho(T, \cdot)$ is defined and fixed on a set of full measure from some point on. Below, if a Lebesgue measurable measure class preserving bijection $\theta: Y \rightarrow Y$ is defined only on a subset $Y \subseteq X$ of full measure, we will allow ourselves to apply $\varphi_{\mathrm{LM}}$ to $\theta$, by identifying $\theta$ with the Lebesgue measurable bijection $\bar{\theta}: X \rightarrow X$ that acts identically on $X \backslash Y$.

Let $\left(T_{\alpha}: \alpha<\kappa\right)$ be an enumeration of $H$. Let

$$
H_{\alpha}=\left\langle T_{\beta}: \beta<\alpha\right\rangle,
$$

where as usual $H_{0}=\{I\}$. We decompose the space $X$ into disjoint pieces $\left(X_{\alpha}\right.$ : $\alpha<\kappa)$, such that $\mu\left(X_{0}\right)=1$, and $\left|X_{\alpha}\right|=2^{\aleph_{0}}$ for all $\alpha<\kappa$. For $\alpha<\kappa$, we will inductively define Lebesgue measurable sets $Y_{\alpha} \subseteq X$ and actions $\rho_{\alpha}: H_{\alpha} \times Y_{\alpha} \rightarrow Y_{\alpha}$ by Lebesgue measurable bijections such that

(1) $Y_{0}=X_{0}$,

(2) $\mu\left(Y_{\alpha}\right)=1$,

(3) $\beta<\alpha \Longrightarrow Y_{\beta} \subseteq Y_{\alpha}$,

(4) $\beta<\alpha \Longrightarrow \rho_{\alpha}\left\lceil H_{\beta} \times Y_{\beta}=\rho_{\beta}\right.$,

(5) $\left(\forall T \in H_{\alpha}\right) \varphi_{\mathrm{LM}}\left(\rho_{\alpha}(T, \cdot)\right)=T$,

(6) $\alpha<\gamma \Longrightarrow Y_{\alpha} \cap X_{\gamma}=\emptyset$.

If we succeed in doing so, then $\rho: H \times X \rightarrow X$ defined by

$$
\rho(T, x)= \begin{cases}\rho_{\alpha}(T, x) & \text { if } T \in H_{\alpha}, x \in Y_{\alpha}, \\ x & \text { otherwise }\end{cases}
$$

is as required. So suppose $\rho_{\gamma}$ and $Y_{\gamma}$ have been defined for all $\gamma<\alpha$. 
Case 1. $\alpha$ is a limit ordinal.

Then we let $Y_{\alpha}=\bigcup_{\gamma<\alpha} Y_{\gamma}$ and

$$
\rho_{\alpha}=\bigcup_{\gamma<\alpha} \rho_{\gamma} .
$$

Case 2. $\alpha=\gamma+1$.

Let $\theta: Y_{\gamma} \rightarrow Y_{\gamma}$ be a bijection such that $\varphi_{\mathrm{LM}}(\theta)=T_{\gamma}$. Then by Lemma 3.3 there is some $Z \subseteq Y_{\gamma}$ such that $\mu(Z)=1$ and $Z$ is invariant under $\theta$ and $\rho_{\gamma}(T, \cdot)$ for all $T \in H_{\gamma}$, and an action $\rho^{0}: H_{\alpha} \times Z \rightarrow Z$ such that $\rho^{0}\left(T_{\gamma}, \cdot\right)=\theta\lceil Z$ and

$$
\rho^{0} \uparrow H_{\gamma} \times Z=\rho_{\gamma} \uparrow H_{\gamma} \times Z
$$

Using Lemma 3.4 we may find an injection $\psi: Y_{\gamma} \backslash Z \rightarrow\left(2^{\mathbb{N}}\right)^{H_{\alpha}}$ such that

$$
\psi\left(\rho_{\beta}(T, x)\right)=\beta(T, \psi(x))
$$

for all $x \in Y_{\gamma} \backslash Z$, where $\beta$ denotes the shift-action of $H_{\alpha}$ on $\left(2^{\mathbb{N}}\right)^{H_{\alpha}}$. Extend $\psi$ to a bijection $\bar{\psi}: W \cup Y_{\gamma} \backslash Z \rightarrow\left(2^{\mathbb{N}}\right)^{H_{\alpha}}$ for some $W \subseteq X_{\gamma+1}$ such that $\bar{\psi}\left\lceil Y_{\gamma} \backslash Z=\psi\right.$, and define $\rho^{1}: H_{\alpha} \times W \cup Y_{\gamma} \backslash Z \rightarrow W \cup Y_{\gamma} \backslash Z$ by

$$
\rho^{1}(T, x)=\bar{\psi}^{-1}(\beta(T, \bar{\psi}(x))) .
$$

If we then let

$$
\rho_{\alpha}=\rho^{0} \cup \rho^{1}
$$

and $Y_{\alpha}=Y_{\gamma} \cup W$, then (1)-(6) above are satisfied.

The proof of the category case is similar.

\section{Open problems}

Throughout this section, $(X, \mu)$ will denote a standard Borel probability space.

In light of the results of this paper, as well as the strong analogy between the lifting problem for $\operatorname{Aut}(X, \mu)$ (Question 1.1) and the lifting problem for the measure algebra, there are several open problems that suggest themselves. Surely the most important open problem is the following:

Problem 4.1. Is it consistent with ZFC that Id : $\operatorname{Aut}(X, \mu) \rightarrow \operatorname{Aut}(X, \mu)$ does not split, i.e. that there is no homomorphic lifting $h: \operatorname{Aut}(X, \mu) \rightarrow G_{\mathrm{mp}}(X, \mu)$ ?

The analogous question for the measure algebra was settled in the affirmative by Shelah, 18 and 19, and so one naturally expects that Problem 4.1 also has a positive answer. This view seems to be further supported by the result of Glasner, Tsirelson and Weiss [7] that no non-trivial Borel measure preserving near-action of Aut $(X, \mu)$ on a standard Borel probability space can have a Borel spatial model.

It is worthwhile pointing out that the following naïve approach to Problem 4.1 does not work: Let $\lambda$ denote the Lebesgue measure on $\mathbb{R}$. For each $A \subseteq[0,1)$ Borel, associate to it the measure preserving bijection $T_{A}:[0,2) \rightarrow[0,2)$ given by

$$
T_{A}(x)= \begin{cases}x+1 & \text { if } x \in A, \\ x-1 & \text { if } x-1 \in A, \\ x & \text { otherwise }\end{cases}
$$

Then for each $[A] \in \operatorname{MALG}([0,1), \lambda)$ this defines a unique element of $\bar{\varphi}\left(T_{A}\right)$ in $\operatorname{Aut}([0,2), \lambda)$, and if we consider $\operatorname{MALG}([0,1), \lambda)$ as a group under the symmetric difference operation, then $[A] \mapsto \bar{\varphi}\left(T_{A}\right)$ is a group homomorphism. 
One might now have hoped that if there was a lifting of $\operatorname{Aut}([0,2), \lambda)$, then the above construction would lead to a lifting of MALG $([0,1), \lambda)$. Ilijas Farah has pointed out that this is not so, because as a group, MALG $([0,1), \lambda)$ always has a lifting. This may be seen, for instance, by an easy induction.

The failure of this approach does prompt the following question:

Problem 4.2. What is the relationship between the lifting problem for $\operatorname{Aut}(X, \mu)$ and the lifting problem for $\operatorname{MALG}(X, \mu)$ ? In particular, does a lifting for one imply a lifting for the other?

The proof of Glasner, Tsirelson and Weiss relies on the fact that the spatial model implementing the given near-action is (jointly) Borel, i.e. that the spatial action $\rho: G \times X \rightarrow X$ is Borel as a map from $G \times X$ to $X$. The following natural question was brought up by Solecki:

Problem 4.3. Does it follow from $\mathrm{CH}$ that every near-action has a separately Borel spatial model, i.e. a pointwise implementing action $\rho: G \times X \rightarrow X$ such that for all $x \in X$ the map $g \mapsto \rho(g, x)$ is Borel, and for all $g \in G$ the map $x \mapsto \rho(g, x)$ is Borel?

The proof of Theorem 1.2 does not seem to give any information about this. Solecki's question also brings up the more general question of what sort of regularity properties are necessary for the Glasner-Tsirelson-Weiss result to go through. As a "test case" it would be of interest to examine Problem 4.1 from the point of view of $\mathrm{AD}$, the Axiom of Determinacy, since it is a well-known fact that AD implies that all subsets of the reals have all common regularity properties; see e.g. [10, Ch. 33].

Problem 4.4. Does it follow from AD that there is no homomorphic lifting $h$ : $\operatorname{Aut}(X, \mu) \rightarrow G_{\mathrm{mp}}(X, \mu)$ ? More generally, does it follow from AD that no Levy group can act pointwise in a non-trivial measure preserving way?

Another question that arises from the proof of Theorem 1.6 is the problem of Borel hierarchy complexity of the automorphisms constructed for the lifting. It is clear that nothing in the construction produces a bound on the rank in the Borel hierarchy of the Borel bijections produced. So we ask:

Problem 4.5. Is it consistent with ZFC to have a lifting $h: \operatorname{Aut}(X, \mu) \rightarrow G_{\mathrm{mp}}(X, \mu)$ such that for some $\gamma<\omega_{1}, h(T) \in \Pi_{\gamma}^{0}$ for all $T \in \operatorname{Aut}(X, \mu)$ ? That is, can we produce a lifting where all the Borel automorphisms have a bounded Borel rank? If yes, does the existence of such a lifting follow from $\mathrm{CH}$ ?

The problem is analogous to a problem of A.H. Stone, see Problem DO.c in [5, which asks the same for the lifting problem for $\operatorname{MALG}(X, \mu)$. This is (to my knowledge) still open for $\operatorname{MALG}(X, \mu)$.

As mentioned in the introduction, Carlson, Frankiewicz and Zbierski have shown that if we add $\aleph_{2}$ Cohen reals (or random reals) to a model of $\mathrm{CH}$, then $\operatorname{MALG}(X, \mu)$ still has a lifting into $\mathcal{B}(X)$. In particular, it is consistent with $\neg \mathrm{CH}$ to have a lifting of $\operatorname{MALG}(X, \mu)$. We ask analogously:

Problem 4.6. Is it consistent with $\neg \mathrm{CH}$ to have a lifting of $\operatorname{Aut}(X, \mu)$ ? In particular, if we add $\aleph_{2}$ Cohen reals (or random reals) to a model of $\mathrm{CH}$, do we still have a lifting of $\operatorname{Aut}(X, \mu)$ ? 
Our next problem concerns Theorem 3.1. In [15], Maharam shows that there always is a lifting of $\operatorname{MALG}(X, \mu)$ with Lebesgue measurable sets. (Maharam attributes this result to von Neumann.) In light of Theorem 3.1, we therefore ask:

Problem 4.7. Does it follow from ZFC alone that there is a Lebesgue measurable lifting of $\operatorname{Aut}(X, \mu)$ ?

Finally, David Fremlin has asked the following questions, to which this author does not know the answer.

Problem 4.8. In Theorem[1.6, can we dispense with the hypothesis of $\mathcal{J}$ containing at least one uncountable Borel set? In particular, does Theorem 1.6 hold if we take $\mathcal{J}$ to be the set of all countable subsets of $X$ ?

Fremlin's next question concerns semigroups rather than groups. If $X$ is a Polish space, $\mathcal{J}$ a $\sigma$-ideal of $\mathcal{B}(X), \mathfrak{A}=\mathcal{B}(X) / \mathcal{J}$ and $G$ is a countable semigroup of Boolean homomorphisms from $\mathfrak{A}$ to itself, then in [6, 344B], it is shown that $G$ can be represented by a family $f_{\pi}$ of Borel functions from $X$ to itself such that $f_{\pi \phi}=f_{\phi} f_{\pi}$ for all $\pi, \phi \in G$. Fremlin asks:

Problem 4.9. Does this theorem hold for semigroups $G$ of cardinality $\aleph_{1}$ ?

\section{ACKNOWLEDGEMENTS}

The author would like to thank David Fremlin for carefully reading a very preliminary version of this paper, and for pointing out the right generality in which the main theorem should be stated. He would also like to thank Katherine Thompson for many useful discussions about the ideas for this paper.

\section{REFERENCES}

1. T. Carlson, R. Frankiewicz, and P. Zbierski, Borel liftings of the measure algebra and the failure of the continuum hypothesis, Proceedings of the American Mathematical Society 120 (1994), 1247-1250. MR1176066 (94f:03058)

2. I. Farah, Analytic quotients: theory of liftings for quotients over analytic ideals on the integers, Memoirs of the American Mathematical Society 148 (2000), no. 702. MR.1711328 (2001c:03076)

3. , Rigidity conjectures, Lecture Notes in Logic, no. 19, Association for Symbolic Logic, 2005. MR2143881 (2006j:03062)

4. All automorphisms of the Calkin algebra are inner, to appear in Annals of Math.

5. D. Fremlin, Problems, http://www.essex.ac.uk/maths/staff/fremlin/problems.pdf.

6. , Measure theory, vol. 3. Measure algebras (2nd printing), Torres Fremlin, 2004. MR2459668

7. E. Glasner, B. Tsirelson, and B. Weiss, The automorphism group of the Gaussian measure cannot act pointwise, Israel Journal of Mathematics 148 (2005), 305-329. MR2191233 (2006i:37008)

8. E. Glasner and B. Weiss, Spatial and non-spatial actions of Polish groups, Ergodic Theory and Dynamical Systems 25 (2005), 1521-1538. MR2173431 (2006h:37003)

9. P. Halmos, Lectures on ergodic theory, Chelsea Publishing Co., 1956. MR.0097489 (20:3958)

10. T. Jech, Set theory, Springer Monographs in Mathematics, Springer-Verlag, 2003. MR1940513 (2004g:03071)

11. A. Kechris, Classical descriptive set theory, Graduate Texts in Mathematics, no. 156, SpringerVerlag, 1995. MR.1321597 (96e:03057)

12. K. Kunen, Set theory, an introduction to independence proofs, Studies in Logic and the Foundations of Mathematics, no. 102, North-Holland Publishing Co., 1980. MR.597342 (82f:03001)

13. A. Kwiatkowska and S. Solecki, Spatial models of boolean actions and groups of isometries, to appear in Ergodic Theory and Dynamical Systems. 
14. G.W. Mackey, Point realizations of transformation groups, Illinois Journal of Mathematics 6 (1962), 327-335. MR0143874 (26:1424)

15. D. Maharam, On a theorem of von Neumann, Proceedings of the American Mathematical Society 9 (1958), 987-994. MR0105479 (21:4220)

16. Y. N. Moschovakis, Descriptive set theory, North-Holland Publishing Co., 1980. MR.561709 (82e:03002)

17. C.N. Phillips and N. Weaver, The Calkin algebra has outer automorphisms, Duke Mathematics Journal 139 (2007), no. 1, 185-202. MR2322680 (2009a:46123)

18. S. Shelah, Lifting problem of the measure algebra, Israel Journal of Mathematics 45 (1983), 90-96. MR710248 (85b:03092)

19. _ Proper and improper forcing, second ed., Springer-Verlag, 1998. MR.1623206 (98m:03002)

20. S. Shelah and J. Steprans, PFA implies all automorphisms are trivial, Proceedings of the American Mathematical Society 104 (1988), 1220-1225. MR935111 (89e:03080)

21. B. Velickovic, $O C A$ and automorphisms of $\mathcal{P}(\omega) /$ fin, Topology and its Applications 49 (1993), no. 1, 1-13. MR1202874 (94a:03080)

22. J. von Neumann and M.H. Stone, The determination of representative elements in the residual classes of a boolean algebra, Fundamenta Mathematicae 25 (1935), 353-378.

Kurt Gödel Research Center, University of Vienna, Währinger Strasse 25, 1090 Vienna, Austria

E-mail address: asger@logic.univie.ac.at 